\title{
Pädiatrische Notfälle
}

\section{Bei Kindern kann der Wolf im Schafspelz erscheinen}

\author{
Kinder sind keine kleinen Erwachsenen. Pädiatrische Notfälle stellen \\ deshalb für den Hausarzt immer eine besondere Herausforderung dar. \\ Hinter einem zunächst harmlos anmutenden Symptom kann sich eine \\ lebensbedrohliche Erkrankung verbergen.
}

- Bei einem zweijährigen Mädchen tritt plötzlich hohes Fieber bis $40,1^{\circ} \mathrm{C}$ mit leichtem Husten und diffusen Bauchschmerzen auf. Klinisch ist das Abdomen unauffällig. Laborchemisch sind die Entzündungsparameter deutlich erhöht. Der Röntgenthorax zeigt eine Unterlappenpneumonie.

„Dieser Fall zeigt, dass sich bei Kindern eine Pneumonie auch sehr untypisch, nämlich als akuter Bauchschmerz präsentieren kann", so Prof. Urs Frey, Basel. $\mathrm{Zu}$ den klassischen Symptomen einer kindlichen Pneumonie zählen Tachypnoe, Zyanose, stoßende Atmung, Einziehungen, Nasenflügeln, Husten, Thoraxschmerzen und asymmetrische Thoraxbewegungen.

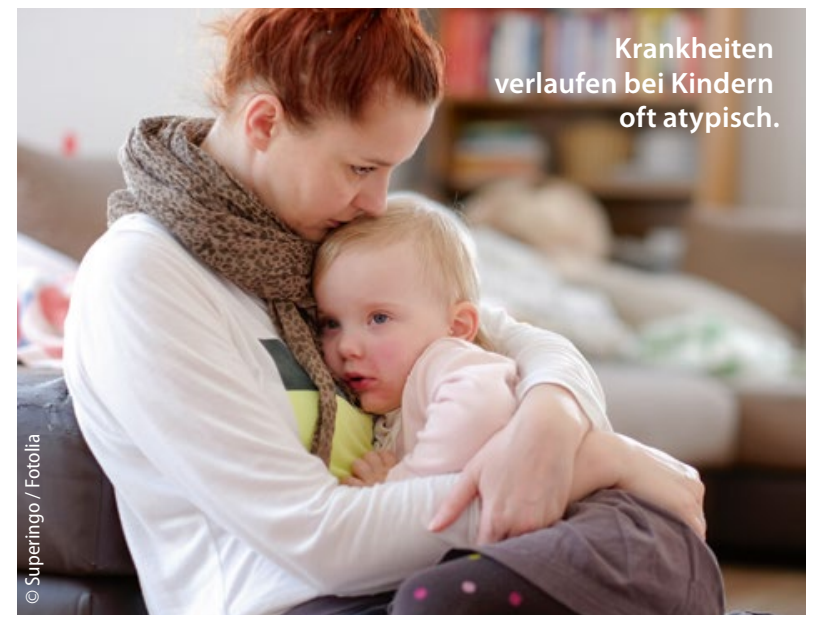

Nicht immer einfach ist die Frage zu beantworten, ob sich hinter dem „Wheezing" eine infektinduzierte Bronchitis bzw. Bronchiolitis oder ein Asthma bronchiale verbirgt. „Die spastische

ten Lebensjahr, prä- und postnatale Tabakexposition sowie niedriges Geburtsgewicht und Gestationsalter. Typisch für einen Asthmaanfall ist die Atemnot mit verlängertem Exspirium.

\section{Fieber ohne Fokus: V.a. Meningitis}

Entwickelt ein Kleinkind plötzlich hohes Fieber, ohne dass ein Fokus gefunden wird, muss der Verdacht auf eine bakterielle Meningitis bzw. Meningokokkensepsis geäußert und eine sofortige Antibiotikatherapie eingeleitet werden. Neben Röntgenthorax, Urinstatus und Blutkulturen muss schon sofort eine Liquorpunktion durchgeführt werden. Bei unbekanntem Erreger empfiehlt sich die Gabe von Ceftriaxon plus Amoxicillin i.v., bei Streptokokken der Gruppe B Penicillin $\mathrm{G}$ plus Amikacin i.v.

\section{Gastroenteritis oder Gedeihstörung?}

Bei Kindern mit Durchfällen gilt es zunächst das Ausmaß der Dehydrierung abzuschät-

\section{Pfeifende Geräusche: Was steckt dahinter?}

Bei pfeifenden Atemgeräuschen mit in spiratorischem Stridor müssen diskutiert werden: Fremdkörper, Quinckeödem, infektiöses Krupp-Syndrom und bakterielle Laryngotracheobronchitis. Der Krupp wird durch virale Infekte ausgelöst und geht mit verlängertem Inspirium, bellendem Husten, Heiserkeit und Einziehungen einher, häufig besteht Fieber. Therapeutisch empfiehlt sich neben einer Luftbefeuchtung die inhalative oder systemische Gabe von Steroiden. Meist ist der Verlauf gutartig.

Eine bakterielle Laryngotracheobronchitis ist dagegen ein schweres Krankheitsbild mit hohem Fieber, Die Kinder machen einen schwerkranken Eindruck und benötigen immer ein Antibiotikum.
Bronchitis hat ihren Höhepunkt um das erste Lebensjahr, ein Asthma bronchiale manifestiert sich meist später", so Frey. Risikofaktoren für rezidivierende obstruktive Atemwegserkrankungen sind Atopie, häufige Atemwegsinfekte im ers-

\section{Wichtige Fragen bei pädiatrischen Notfällen}

- Telefonisch beurteilen oder Kind sehen?

- Könnte sich die Krankheit atypisch präsentieren?

- Handelt es sich um ein harmloses oder um ein potenziell gefährliches Krankheitsbild?

- Ist eine rasche Verschlechterung möglich?

- Ist meine Kompetenz ausreichend?

- Muss ich das Kind überwachen oder verlegen? zen. Dabei müssen der Bewusstseinszustand, der Hautturgor, die Fontanelle, der Puls, der Blutdruck, die Urinausscheidung und die Atemfrequenz erfasst werden. „Auch wenn die Arbeitshypothese „akute Gastroenteritis“ naheliegt, sollte man auch an eine Gedeihstörung, also eine chronische Erkrankung, die mit einer Malabsorption einhergeht wie eine zystische Fibrose oder eine Zöliakie, denken", so Frey.

Bei unspezifischen Symptomen sind auch seltene Erkrankungen möglich. So fand sich bei einem Kind, das wegen einer Verschlechterung des Allgemeinbefindens mit Appetitlosigkeit, Gedeihstörung und Fieber auffiel, ein Neuroblastom im Mediastinum.

Dr. med. Peter Stiefelhagen

- Quelle: medArt, 25.6.2015 in Basel 